\title{
Determening the Optimum Compromise between SAR Data Compression and Radiometric Performance -An Approach Based on the Analysis of TerraSAR-X Data-
}

\author{
Marwan Younis, Johannes Boer, Carlos Ortega, Daniel Schulze, Sigurd Huber and Josef Mittermayer \\ German Aerospace Center (DLR), Microwaves and Radar Institute \\ Email: marwan.younis@dlr.de
}

\begin{abstract}
Data compression is crucial for modern synthetic aperture radar systems where high resolution or large coverage may result in huge amounts of raw data. Modern satellite systems, such as TerraSAR-X, give complete flexibility in choosing between various compression levels. This, however, results in additional effort to decide on the suitable compression level used, which may depend on the operation mode, polarization, scene backscatter, etc. The paper describes the approach used in the case of TerraSAR-X and shows the result of analyzing the data acquired during the commissioning phase. The methology is considered novel in the sense that it combines SAR measured data analysis with theoretical, i.e. model based simulations, results and later combines theory and measured data to extract optimum compression levels.
\end{abstract}

\section{INTRODUCTION}

A technique commonly used for raw data compression in synthetic aperture radar (SAR) systems is block adaptive quantization (BAQ). The BAQ compression technique is lossy, since the data samples resulting after decompression are not equal to the original input samples. Different variations of BAQ algorithms exist, but all of them can basically be understood as an adaptive scaling and re-quantization of the data, resulting in reduction of the effective data rate. The BAQ compression is modeled as an additive noise term, masking the original SAR raw data. Increasing the compression rate results in an increased quantization noise or equivalently a reduced radiometric resolution.

For TerraSAR-X a real time BAQ compression was implemented in hardware. The BAQ compression levels can be selected between 8:8, 8:6, 8:4, 8:3, and 8:2 where the first digit represents the 8 bit analog-to-digital converter (ADC) quantization, while the second digit is the number of bits per I/Q-sample resulting after BAQ compression. The task was to decide on the compression level appropriate for each operation mode with respect to an allowed performance degradation. These are written in the BAQ setting table. The paper describes the used simulative model based approach for populating the BAQ setting table. This includes deriving the parameters representing the performance of the BAQ. During the commission phase a large number of data takes were analyzed and the results used to verify the the approach on one side, and to adapt the compression level according to the measured degradation.

\section{System AND Signal Model}

In the following the system and signal models representing the quantization and BAQ compression of the input are defined. In order to assess the quality of the signal after BAQ compression, it is required to develop a figure-of-merit based on the statistical relation between the complex input and output signal.

\section{A. System Model}

With reference to Fig. 1, the complex input signal $\grave{x}(t)$ is band limited to $B_{w}$ and then sampled at a rate $f_{s}$ yielding the complex input signal $x\left(t_{k}\right)$. The receiver noise is modeled as additive white Gaussian noise $n\left(t_{k}\right)$ which is uncorrelated to the input signal. At this point the signal-to-noise ratio for the raw SAR echo signal after amplification and down conversion is given by $S N R_{\mathrm{in}}$. Each of the real and imaginary part of the noisy input signal is amplitude limited to $\pm V_{\text {clip }}$ and quantized in the ADC. The resultant complex signal at the output of the ADC $\grave{y}\left(t_{k}\right)$ has one of $2^{b-1}$ amplitude levels, where $b$ is the number of Bits. The BAQ encoding, i.e. lossy data compression, is applied to the output of the ADC yielding data blocks sharing a common scaling factor (also known as exponent) while each sample is represented by its mantissa. The complex output $y\left(t_{k}\right)$ signal results after BAQ decoding. In the following the subscript ${ }_{k}$ indicating the time-discrete nature of the signals will be dropped in favor of a simplified representation.

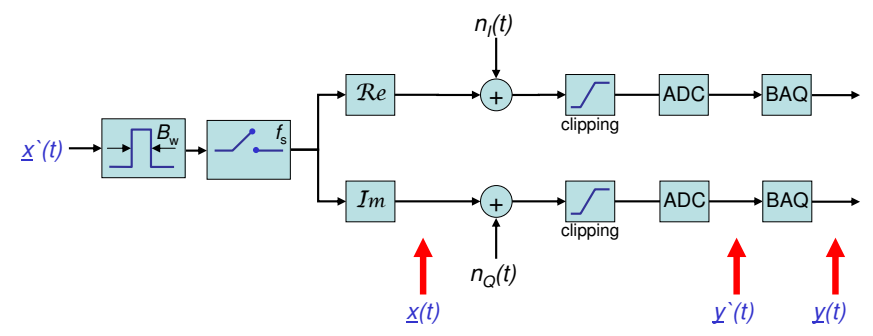

Fig. 1. Block diagram of system model used for the BAQ performance analysis.

Throughout this paper a figure-of-merit will be developed which serves to give a measure of the data quality. The same figure-of-merit can be applied both to the ADC and the BAQ 
outputs. In this sense no distinction is made between quantization and data compression; this way the BAQ compression is viewed as a type of quantization. For compactness only the output of the BAQ will be considered in the following, while the output of the ADC is referred to as BAQ8:8, i.e. no compression.

\section{B. Signal Model}

The input signal is characterized through its autocorrelation function $R_{x x}(\tau)=E\left\{x(t+\tau) x^{*}(t)\right\}$ where the average signal power (apart from a resistive scaling factor) is $R_{x x}(0)=$ $E\left\{|x(t)|^{2}\right\}$. Using similar representation for the receiver noise $n(t)$ results in the following expression for the signal-to-noise ratio at the input of the quantizer

$$
S N R_{\text {in }}=\frac{E\left\{|x(t)|^{2}\right\}}{E\left\{|n(t)|^{2}\right\}}
$$

The signal at the output of the BAQ $y(t)$ is modeled as the sum of the scaled input signal (including receiver noise) in addition to an error contribution due to the quantization and BAQ compression, which is modeled by an additive quantization noise $q(t)$ :

$$
y(t)=A x(t)+A n(t)+q(t)
$$

The quantization noise itself is the sum of the granular and clipping noise. The scaling, represented by the factor $A$, is inherent to the quantization process and is crucial for the correct description of the quantization. In the above expression $A$ is independent of the specific sample value but rather a statistical description of the data and thus $A$ represents the conversion gain.

Similarly as for the input, the output signal is characterized by its autocorrelation function $R_{y y}(\tau)$. Here it is assumed that the quantization noise and the input signal (and receiver noise) are uncorrelated ${ }^{1}$. Specifically the average power of the output signal is of interest, is given by

$$
R_{y y}(0)=A^{2} E\left\{|x(t)|^{2}\right\}+A^{2} E\left\{|n(t)|^{2}\right\}+E\left\{|q(t)|^{2}\right\}
$$

The figure-of-merit requires the quantification of the relation between the input and output signal. Statistically this is characterized by the cross-correlation of the (noise free) input signal $x(t)$ and the BAQ output $y(t)$ give by $C_{x y}(\tau)=$ $E\left\{x(t+\tau) y^{*}(t)\right\}$. Specifically the value at $\tau=0$ is of interest:

$$
C_{x y}(0)=A E\left\{|x(t)|^{2}\right\}
$$

\section{Determining the ADC And BAQ Performance}

In the following the parameters used to quantify the performance of the ADC and BAQ are stated. The "quality" of the input signal is given by its signal-to-receiver-noise ratio $S N R_{\text {in }}$ as defined in (1). At the output we define the signal-to-noise ratio $S N R_{\text {baq }}$ as the ratio of the signal power in the absence

\footnotetext{
${ }^{1}$ For TerraSAR-X with 8-Bit quantization this condition is fulfilled, but in general the assumption of additive independent quantization noise is not necessarily satisfied, see for example [1] for details.
}

of receiver and quantization noise to the sum of quantization plus receiver noise power [2]. This is expressed as:

$$
S N R_{\mathrm{baq}}=\frac{C_{x y}^{2}(0)}{R_{y y}(0) R_{x x}(0)-C_{x y}^{2}(0)}
$$

It should be noted, that the above expression avoids the common mistake often seen in literature which computes the output $S N R$ without considering the conversion gain $A$; This leads to an erroneous expression where, in specific cases, the output $S N R_{\text {baq }}$ can become higher than the input $S N R_{\text {in }}$. An exhaustive investigation of this is beyond the scope of this paper, we just note (5) can be reformulated as:

$$
S N R_{\text {baq }}=\frac{S N R_{\text {in }}}{1+\frac{E\left\{|q(t)|^{2}\right\}}{A^{2} E\left\{|n(t)|^{2}\right\}}}
$$

where clearly $S N R_{\text {baq }} \leq S N R_{\text {in }}$ (see Fig. 2 later for a plot of $S N R_{\text {baq }}$ versus $\left.S N R_{\text {in }}\right)$.

The signal-to-noise ratio is not sufficient to quantify the performance of the BAQ. We introduce the conversion gain as an additional performance parameter. This is readily obtained from (4) to be

$$
A=\frac{C_{x y}(0)}{R_{x x}(0)}
$$

A third quantity is introduced to describe the amount of clipping at the input of the quantizer. This is the signal-toclipping ratio, also known as input $1 \sigma$ signal level below the clipping level, which is defined as:

$$
\gamma_{c l i p}=\frac{\sqrt{R_{x x}(0)}}{V_{c l i p}}
$$

In the above expression $\gamma_{c l i p}$ can be understood as the ratio between the square of the average power of the noise-free input signal to the maximum input range for the ADC.

\section{Performance Parameter Analysis}

In this section the influence of various parameters on the figures-of-merit derived in the previous section is analyzed. The analysis is performed by simulations based on the system and signal models given earlier in section II. This analysis is the basis for determining the appropriate BAQ operation settings for TerraSAR-X namely the receiver gain and BAQ compression level.

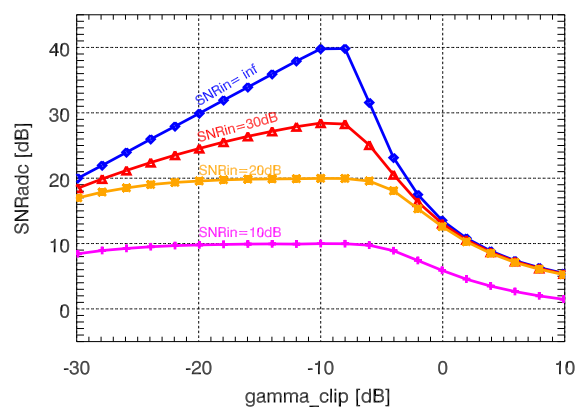

Fig. 2. Signal-to-noise ratio at ADC output (disregarding the BAQ). 
In principal performance analysis is straightforward. For clarity we start by disregarding the BAQ compression, i.e. for BAQ 8:8, and analyze the influence of $S N R_{\text {in }}$ and $\gamma_{c l i p}$. Fig. 2 shows the signal-to-noise ratio at the ADC output as a function of $\gamma_{c l i p}$ with $S N R_{\text {in }}$ as a parameter. It is seen that the best performance for a noiseless input signal is at $\gamma_{\text {clip }}=-8.88 \mathrm{~dB}$; however even for noisy input signals the optimum $\gamma_{\text {clip }}$ does not deviate much from this value. Further, for any $S N R_{\text {in }}$ the degradation is higher for $\gamma_{c l i p}>-8.88 \mathrm{~dB}$ then when $\gamma_{\text {clip }}<-8.88 \mathrm{~dB}$; thus, the influence of clipping noise is more severe than the granular noise.

For TerraSAR-X, where no automatic gain control is available, the gain setting is preset to a fixed value commanded for each data take. With this constraint it seems preferable to set $\gamma_{\text {clip }}$ to values smaller than $-8.88 \mathrm{~dB}$, thus avoiding clipping the raw data at the expense of a (slight) degradation in NESZ value (see [3] for an investigation on this point).

Next, the influence of the various BAQ compression levels on the performance is investigated. Fig. 3(a) shows $S N R_{\text {baq }}$ versus $S N R_{\text {in }}$ for different BAQ levels when the clipping level is optimum $\gamma_{c l i p}=-8.88 \mathrm{~dB}$. To get a quantitative measure of influence of the BAQ level, the degradation of $S N R_{\text {baq }}$ for the $i$-th level (i.e. BAQ 8:i) given by $S N R_{\text {adc }}-S N R_{b a q 8: i}$ is shown in Fig. 3(b) versus $S N R_{\text {in }}$. This plot can be used to set the BAQ level depending on the input power level.

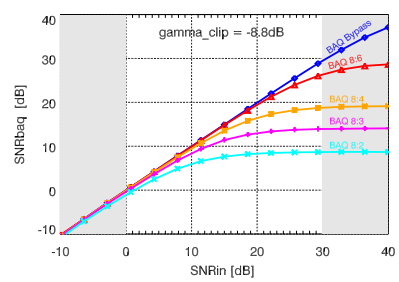

(a) signal-to-noise ratio $S N R_{\text {baq }}$

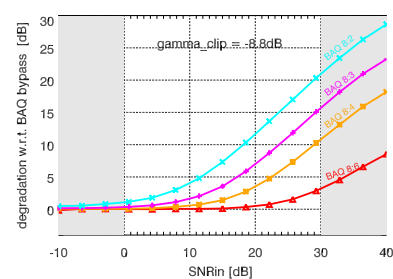

(b) degradation in $S N R_{\text {baq }}$
Fig. 3. BAQ Performance versus $S N R_{\text {in }}$ with BAQ level as a parameter and optimum $\gamma_{c l i p}=-8.8 \mathrm{~dB}$.

Clearly $S N R_{\text {baq }}$ degrades as the BAQ compression is increased; this is an expected result, since the BAQ is a lossy compression technique. An interesting effect can be seen from the Fig. 3(b), where the degradation becomes smaller for decreasing $S N R_{\text {in }}$ values, which is true for all BAQ compression levels.

Last the effect of clipping and BAQ compression on the conversion gain is investigated. The conversion gain versus clipping is shown in Fig. 4 with the BAQ compression level as a parameter. It is seen that the gain drops when $\gamma_{\text {clip }}>$ $-8.8 \mathrm{~dB}$. Comparing Fig. 4(a) to Fig. 4(b) it is concluded that the influence of $S N R_{\text {in }}$ on the conversion gain is marginal, provided that $\gamma_{\text {clip }} \leq-8.8 \mathrm{~dB}$.

An observation made from Fig. 4 is that the conversion gain shows a dependence on the BAQ compression. This effect is a result of the specific BAQ algorithm used for TerraSAR-X. If uncorrected, this would lead to an error in the radiometric calibration, which depends on the BAQ compression level.
However, since the variation of the conversion gain is nearly independent of $\gamma_{c l i p}$ it is straightforward to compensate the gain variation by introducing a BAQ dependent (but signal independent) compensation factor.

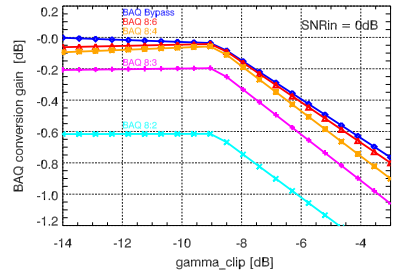

(a) $S N R_{\mathrm{in}}=0 \mathrm{~dB}$

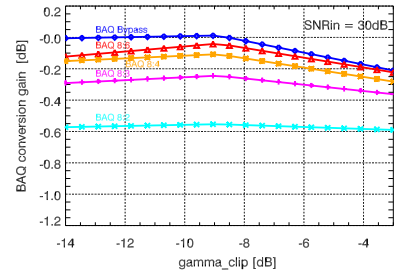

(b) $S N R_{\text {in }}=30 \mathrm{~dB}$
Fig. 4. conversion gain versus clipping level $\gamma_{c l i p}$ with BAQ compression as a parameter.

\section{BAQ TABLE GENERATOR FOR TERRASAR-X}

The last section showed the effect dictating the approach for deciding on the appropriate compression level. This can be formulated as follows: for any allowable degradation an increased noise level of the input signal results in a higher possible compression rate, i.e. less bits are required for quantization. Based on this, a procedure for populating the BAQ setting table was developed and implemented specifically for TerraSAR-X. The table generator uses the results of the BAQ performance (c.f. section IV) together with the $N E S Z$ values provided from the TerraSAR-X performance estimator [4] in order to determine the BAQ setting for each operation mode, polarization, channel and beam. The basic aproach is to allow a upper limit of NESZ degradation due to the BAQ. As shown in section IV this degradation is dependent on the actual input $S N R$ values. Thus a link must be established between NESZ and $S N R_{\text {in }}$ values, which is found to be through the Radar Cross Section. Finally, the BAQ table setting is such as to ensure that $90 \%$ of the RCS values of the earths land mass result in an NESZ degradation of less than $2 \mathrm{~dB}$.

\section{Analysing the Measured Data}

In this section we show the results of analyzing the TarraSAR-X data in order to confirm the approach used for calculating the BAQ table settings on one side and to assess the anticipated degradation values on the other. When analyzing real measured data, not all quantities are accessible as is the case in a simulation model. Specifically in the case of measurements, the data after the BAQ can not be used to determine the actual value of the quantization error (otherwise it would be an easy task to correct for the quantization error). Consequently two verification approaches are possible:

1) Acquire BAQ8:8 data and reprocess the data for all $B A Q$ levels to compute the the quantization error.

2) Use a combination between measured data and model based results to statistically verify the BAQ degradation.

Although both approaches were followed in the case of TerraSAR-X, we confine to the second of the above two approaches. 
We start by analyzing the signal-to-clipping ratio. In order to compare simulation results to measurements we compute the simulated signal-to-clipping ratio based on the noisy output signal $y(t)$ according to $\gamma_{\text {clip }}=\sqrt{R_{y y}(0)} / V_{\text {clip }}$ instead of using (8); this is actually independent of $S N R_{\text {in. }}$ Fig. 5 shows the percentage data clipping versus signal-to-clipping ratio both from the simulation ${ }^{2}$ and using the analyzed data from a total of 1450 TerraSAR-X data takes (equivalent to about 630000 statistical samples on measurement data). The measurement results follow the trend of the simulation curve and show a very good coincidence for low clipping levels, where $\gamma_{\text {clip }}<-8.8 \mathrm{~dB}$ which is the relevant range. The results presented in Fig. 5 were the basis for the receiver gain setting as detailed in [3].

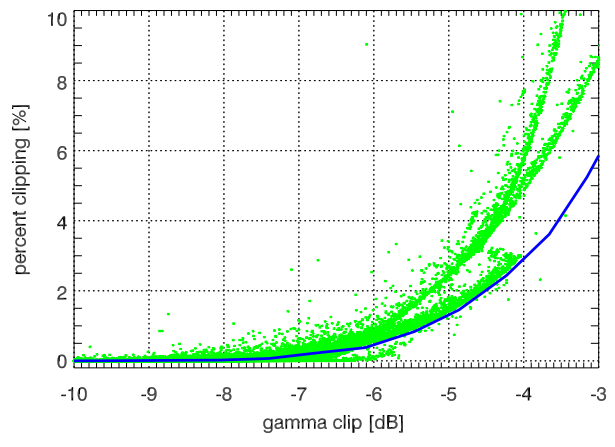

Fig. 5. Percentage clipping as a function of signal-to-clipping ratio both from simulation and measured TerraSAR-X data.

Next an analysis of the BAQ degradation is due. TerraSAR-X data takes provide two types of signals relevant for the BAQ analysis. One is the complex raw SAR signal, which, using the model of section II, is characterized by $R_{y y}(\tau)$. The second are noise measurements obtained by acquiring data without turning the transmitter on. Applying the previous model these noise measurements represent the quantized version $\tilde{n}(t)$ of the actual receiver noise with autocorrelation function $R_{\tilde{n} \tilde{n}}(\tau)$. The expression for the measured signal-to-noise ratio $S N R_{\mathrm{tsx}}$ then is:

$$
\begin{aligned}
S N R_{\mathrm{tsx}} & =\frac{R_{y y}(0)}{R_{\tilde{n} \tilde{n}}(0)}-1 \\
& =\frac{A^{2} E\left\{|x(t)|^{2}\right\}+E\left\{|q(t)|^{2}\right\}-E\left\{|\grave{q}(t)|^{2}\right\}}{A^{2} E\left\{|n(t)|^{2}\right\}+E\left\{|\grave{q}(t)|^{2}\right\}}
\end{aligned}
$$

where $\grave{q}(t)$ represents the quantization noise of the noise measurement with BAQ8:8, while $q(t)$ is the SAR signal quantization noise. Note that the first line in the above equation includes only quantities accessible through the measurements ${ }^{3}$. As commented earlier it is not possible to extract the quantization noise from the measured data, thus the above formulation can only be used in conjunction with a model, i.e. a BAQ

${ }^{2}$ For a known $p d f$ the percentage clipping can actually be computed analytically, see [5] for example.

${ }^{3}$ The equivalent expression to for $S N R_{\mathrm{tsx}}$ in terms of the quantities introduced in the system model is given by $\frac{R_{y y}(0) R_{x x}(0)}{R_{\grave{y} \grave{y}}(0) R_{x x}(0)-R_{x \grave{y}}^{2}(0)}-1$. signal model, in order to yield information about the BAQ influence.

Thus we chose to combine the measurements of a set of data takes sharing common properties with the simulation model in order to compute the BAQ degradation. The results for the full performance beams of the StripMap and SpotLight operation modes are shown Fig. 6 and Fig. 7 respectively. The left plots in the figures show $S N R_{\text {in }}$ histogram as computed from the measurement data; in the plots the $90 \% S N R_{\text {in }}$ occurrence values are marked. The performance degradation computed for the respective $90 \% S N R_{\text {in }}$ values are shown in the right plots of the figures. The plots on the right also show the percentage occurrences of the different BAQ compression levels. It is seen that the most frequent compression levels have a degradation $\leq 2 \mathrm{~dB}$, which is the allowed level.

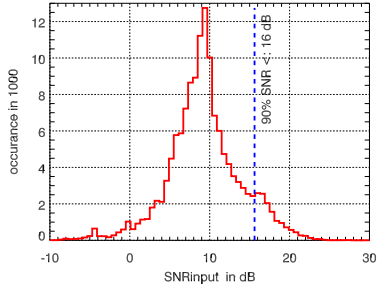

(a) signal-to-noise ratio histogram

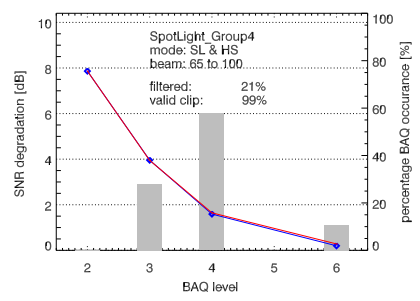

(b) degradation and BAQ occurrence
Fig. 6. Performance curves for the full performance SpotLight beams computed from the measured data.

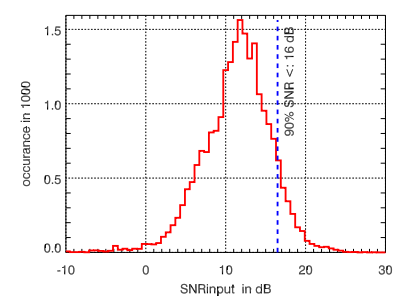

(a) signal-to-noise ratio histogram

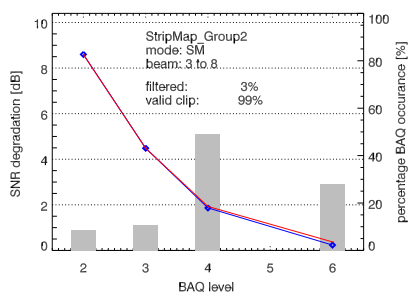

(b) degradation and BAQ occurrence
Fig. 7. Performance curves for the full performance StripMap beams computed from the measured data..

\section{REFERENCES}

[1] B. Widrow, I. Kollár, and M.-C. Liu, "Statistical theory of quantization," IEEE Transactions on Instrumentation and Measurement, vol. 45, no. 2, pp. 353-361, Apr. 1996.

[2] T. Misra and A. Moreira, "Simulation and performance evaluation of the real-time processor for the E-SAR system of DLR," German Aerospace Center, Microwaves and Radar Institute," Tech. Note, 1991.

[3] J. Mittermayer, B. Schättler, and M. Younis, "TerraSAR-X commissioning phase execution and results," in Proc. Int. Geoscience and Remote Sensing Symposium IGARSS'08, Bosten, MA, July 2008.

[4] J. Mittermayer, U. Steinbrecher, A. Meta, N. Tous-Ramon, S. Wollstadt, M. Younis, J. Marquez-Martinez, D. Schulze, and C. Ortega-Miguez, "TerraSAR-X instrument, SAR system performance and command generation," in Proc. European Conference on Synthetic Aperture Radar EUSAR'08, Friedrichshafen, Germany, June 2008.

[5] D. Dardari, "Joint clip and quantization effects characterization in OFDM receivers," IEEE Transactions on Circuits and Systems, vol. 53, no. 8, pp. 1741-1748, Aug. 2006. 